\title{
Sind wir als Zahnärzte davon überhaupt betroffen?
}

\begin{abstract}
Werfen wir einen Blick auf den Alltag in der Zahnarztpraxis jenseits aller politischen Implikationen der neuen Gebührenordnung für Ärzte (GOÄ): Ja, wir Zahnärzte sind Tag für Tag von vielen Gebührennummern der GOÄ betroffen, sogar die grundlegendsten Leistungen der Beratung und Untersuchung befinden sich in der GOÄ.
\end{abstract}

Der Zahnarzt darf und muss nach $₫ 6$ Abs. 2 der Gebührenordnung für Zahnärzte (GOZ) auf bestimmte Kapitel der derzeit für die Privatabrechnung gültigen GOÄ von 1996 zurückgreifen. Dies betrifft neben der Berechnung von Untersuchungsund Beratungsleistungen vor allem zahnärztliche Röntgenleistungen. Daneben greift der Zahnarzt aber selbstverständlich auch zu auf viele kleine und große chirurgische Maßnahmen und diverse Leistungen wie zum Beispiel Berichte, Konsile, Abstriche und vieles mehr. $\$ 6$ Abs. 2 der GOZ legt konkret fest, welche Leistungen der Zahnarzt unter Zugrundelegung der GOÄ berechnen kann. Hierbei ist die Gebührenordnung für Ärzte in der jeweils geltenden Fassung anzuwenden. Wenn also tatsächlich in naher Zukunft die reformierte GOÄ Realität wird, so wirkt sich das sofort und unmittelbar auf alle nach der GOÄ berechneten Leistungen in der Zahnarztpraxis aus.

\section{Beratungsleistungen nach GOÄ}

Alltäglich rechnen wir Zahnärzte die Beratungsleistungen nach der GOÄ ab. Jede einzelne Beratung wird nach der Nummer 1 der GOÄ liquidiert, eine eigene zahnärztliche Beratungsleistung existiert einfach nicht. Auch die weitergehende Beratung nach der GOÄ-Nr. 3 wird alltäglich in der Zahnarztpraxis erbracht. Diese GOÄ-Nummern und ihre Berechnungsmodi finden sich sogar expressis verbis in den allgemeinen Bestimmungen des Kapitels A der GOZ wieder. Ebenso alltäglich rechnen wir Zahnärzte unsere symptombezogenen Untersuchungsleistungen nach der GOÄ-Nr. 5 ab. Eine eigene zahnärztliche symptombezogene Untersuchungsleistung existiert ebenfalls nicht.

Wird das stomatognathe System vollständig untersucht, so fällt die GOÄ-Nr. 6 an, werden aufwendige Fremdanamnesen erhoben oder Bezugspersonen instruiert, so fällt die GOÄ-Nr. 4 an. Jeder Besuch, gegebenenfalls unter welchen Zuschlagsbedingungen auch immer, jedes Konsil, jede kurze Bescheinigung, jeder Arztbrief, jedes medizinische Gutachten kann nur nach der GOÄ berechnet werden (vgl. z.B. GOÄ-Nrn. 48, 50, 60, 70, 75, $80,85)$.

\section{Angrenzende Fachgebiete der Chirurgie}

Selbstverständlich greift die zahnärztliche Chirurgie in die angrenzenden chirurgischen Fachgebiete ein, sodass beispielsweise die Berechnungen von Leistungen an der Kieferhöhle (GOÄNr. 1465 - 1485), Wundversorgungen (GOÄ-Nrn. 2000 - 2010) oder der Chirurgie der Körperoberfläche (Abszesseröffnungen GOÄ-Nrn. 2428, 2430; Hautlappenplastik GOÄ-Nr. 2381, 2382; Probeexzisionen und Exzisionen GOÄ-Nrn. 2401-2404 und vie- les mehr) in praktisch allen Zahnarztpraxen - wenn auch in deutlich unterschiedlichem Umfang - anfallen.

Aus dem Kapitel der MKG-Chirurgie (GOÄ L IX) fallen neben den typisch kieferchirurgischen Operationsleistungen auch für die Zahnarztpraxen häufig kleinere chirurgische Maßnahmen wie die Zahnreposition (GÖ̈-Nr. 2685), das Anlegen von Drahthäkchen (GOÄ-Nr. 2697) oder die Verbandsplatte nach GOÄ-Nr. 2700) immer wieder an. Ebenso spielen einfache Laboratoriumsuntersuchungen zum Beispiel des Speichels oder von Bakterien aus dem Kapitel M der GOÄ sowie die Abstrichentnahme hierfür (GOÄ-Nr. 298) eine Rolle in Prophylaxe und Parodontologie.

\section{Röntgenleistungen sind alltäglich}

Vor allem aber: Wir Zahnärzte greifen alltäglich auf die Röntgenaufnahmen der Zähne (GOÄ-Nr. 5000) und der Kiefer (GOÄ-Nr. 5004, Orthopantomographie) zu. Schädelübersichtaufnahmen fallen typischerweise in der Kieferorthopädie (KFO) und der Funktionsdiagnostik/Prothetik an. Die Bestimmung des Skelettalters nach der GOÄ-Nr. 5037 gehört zu den Aufgaben der KFO. Und neben den klassischen Schädelteil- und Nasennebenhöhlen-Aufnahmen (GÖ̈-Nrn. 5095, 5098) erlangt die digitale Volumentomographie (DVT-Diagnostik) immer größere Bedeutung, die nach den GOÄ-Nrn. 5370 und 5377 berechnet wird.

Diese lange Aufzählung beweist die Relevanz der GOÄ für den zahnärztlichen Alltag. Neben allen grundsätzlichen Erwägungen der Bedeutung der in der vorliegenden Art geplanten Reformierung des freien ärztlichen Gebührenrechts in Deutschland ist allein schon die Häufigkeit der Berechnung von GOÄLeistungen Grund genug, sich in die sich abzeichnende problematische GOÄ-Reform mit aller Kraft einzumischen.

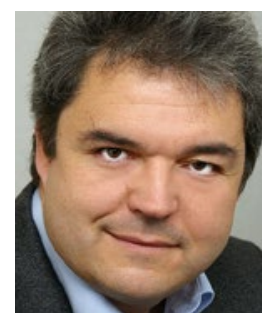

Dr. Dr. Alexander Raff

Mitglied im GOZ-Expertenrat des FVDZ 